\title{
Cigarette smoking alters epithelial apoptosis and immune composition in murine GALT
}

\author{
Stephanie Verschuere ${ }^{1}$, Ken R Bracke ${ }^{2}$, Tine Demoor ${ }^{1}$, Maud Plantinga ${ }^{3}$, Phebe Verbrugghe ${ }^{4}$, Liesbeth Ferdinande ${ }^{1}$, \\ Bart N Lambrecht ${ }^{3}$, Guy GG Brusselle ${ }^{2, *}$ and Claude A Cuvelier ${ }^{1, *}$
}

Smokers have a twofold increased risk to develop Crohn's disease (CD). However, little is known about the mechanisms through which smoking affects CD pathogenesis. Especially Crohn's ileitis is negatively influenced by smoking. Interestingly, the ileum and, more in particular, the Peyer's patches in the terminal ileum are also the sites where the first CD lesions are found. Several chemokines are implicated in the pathogenesis, among which is the CCL20-CCR6 pathway. Here, we studied the gut-associated lymphoid tissue in C57BL/6 wild-type mice and in CCR6-deficient mice after exposure to air or cigarette smoke for 24 weeks. Apoptotic index of the follicle-associated epithelium overlying the Peyer's patches was evaluated. We found that chronic smoke exposure induced apoptosis in the follicle-associated epithelium. Furthermore, immune cell numbers and differentiation along with chemokine expression were determined in Peyer's patches. Important changes in immune cell composition were observed: total dendritic cells, CD4 + T cells (including regulatory $T$ cells) and CD8 $+T$ cells increased significantly after smoke exposure. The CD11b + dendritic cell subset almost doubled. Interestingly, these changes were accompanied by an upregulated mRNA expression of the chemokines CCL9 and CCL20. However, no differences in the increase of dendritic cells were observed between wild-type and CCR6deficient mice. Our results show that cigarette smoke exposure increases apoptosis in the follicle-associated epithelium and is associated with immune cell accumulation in Peyer's patches.

Laboratory Investigation (2011) 91, 1056-1067; doi:10.1038/labinvest.2011.74; published online 2 May 2011

KEYWORDS: apoptosis; cigarette smoke; Crohn's disease; dendritic cell; intestinal epithelium; Peyer's patch

Crohn's disease $(\mathrm{CD})$ is an incapacitating inflammatory disease of the gastrointestinal tract, mainly involving the terminal ileum and colon. The social impact of CD is significant, as it is a chronic and relapsing disorder, which principally affects young patients in the second and third decades of life. ${ }^{1}$ Prevalence ranges from 174 to 210 per 100000 inhabitants in North America and Europe and is still increasing. ${ }^{2,3}$ Despite years of intensive research, ample questions remain on the aetiology of CD. Defects in barrier function as well as alterations in innate and adaptive immune system are involved, triggering an aberrant immune response against gut flora. Although the pathways leading to intestinal inflammation are incompletely understood, there is a wide consensus that both genetic and environmental factors are implicated. ${ }^{4}$

Smoking is the most established environmental risk factor in $\mathrm{CD}$. Moreover, smoking is one of the few reversible risk factors identified hitherto. Current smokers have a twofold higher lifetime risk to develop CD compared with neversmokers. ${ }^{5}$ In addition, smoking is identified as an independent risk factor for the development of clinical, surgical and endoscopic recurrence after surgery. Former smokers have the same recurrence rates as never-smokers, supporting the importance of smoking cessation for CD patients. ${ }^{6}$ Furthermore, smoking is associated with more complicated disease, a higher need for steroids and immunosuppressants and poorer response rate to infliximab. ${ }^{5,7}$

Notwithstanding this substantial epidemiological evidence, little is known about the molecular and cellular mechanisms by which smoking affects the gut and interferes with $\mathrm{CD}$ pathogenesis. Hypotheses include modulation of humoral and cellular immune responses, changes in intestinal cytokine levels, alterations in mucosal blood flow and modifications in

\footnotetext{
'Department of Pathology, Ghent University, Ghent, Belgium; ${ }^{2}$ Laboratory for Translational Research in Obstructive Pulmonary Diseases, Department of Respiratory Medicine, Ghent University Hospital, Ghent, Belgium; ${ }^{3}$ Laboratory of Immunoregulation and Mucosal Immunology, Department of Respiratory Medicine, Ghent University, Ghent, Belgium and ${ }^{4}$ Microbiology and Immunology, School of Biomedical, Biomolecular and Chemical Sciences, University of Western Australia, Perth, Australia

Correspondence: Dr S Verschuere, MD, Department of Pathology, 5 Blok A, University Hospital Ghent, De Pintelaan 185, Ghent 9000, Belgium. E-mail: Stephanie.Verschuere@ugent.be

*These authors contributed equally to this work. 
gut permeability or gut motility by smoke exposure. ${ }^{8}$ However, no single mechanism was able to explain the observed effect of smoking on CD. Previously published human studies investigated distinct anatomical compartments (gut lavage fluid versus peripheral blood mononuclear cells versus colonic mucosa), which makes it difficult to draw general conclusions. ${ }^{9-12}$ On the other hand, established animal models have several limitations to explore the effect of smoking on CD. First, most reports describe the effect of (sub)acute smoke exposure, whereas human smoking usually involves prolonged periods of smoke exposure. ${ }^{13-15}$ Secondly, in several study designs nicotine is administered orally or subcutaneously. ${ }^{16,17}$ Besides the fact that nicotine is not the sole active component in cigarette smoke, oral and subcutaneous administration stand far apart from smoke inhalation. A drawback of both human and animal experimental studies is that they generally focus on changes in the-easier accessible-colon, whereas epidemiological data suggest a smoke-induced increase in Crohn's ileitis rather than colitis. ${ }^{7,8}$

The purpose of this study was to investigate the influence of cigarette smoke on the mucosal immune system of the small intestine in a murine model. Mice were exposed to main stream cigarette smoke for 24 weeks. ${ }^{18}$ Because of the known relationship between smoking and Crohn's ileitis, effects on the gut-associated lymphoid tissue (GALT) of the small intestine-and Peyer's patches (PP) in particular-were examined. Smoking alone is insufficient to cause intestinal inflammation, as observed in both animal models and human individuals. However, in combination with other risk factors, like genetic factors or environmental triggers, it can lead to the development of $\mathrm{CD}$ and therefore we were interested in subclinical effects of smoke exposure in the gut, before full-blown inflammation. We hypothesized that cigarette smoke alters the immune regulation in PP, predisposing the gut to develop $\mathrm{CD}$. Therefore, we first investigated the effect of smoke exposure on apoptosis in the follicleassociated epithelium (FAE) overlying PP. Secondly, we analysed cellular composition and size of PP and mesenteric lymph nodes. Furthermore, expression of chemokines was examined to elucidate how recruitment of immune cells towards the PP is influenced by cigarette smoke. Finally, the role of the CCL20-CCR6 signalling pathway was further investigated by means of CCR6 knockout (KO) mice. We show that smoking alters apoptosis in the FAE and the immune cell composition of PP, which may explain the higher susceptibility of smokers to develop CD.

\section{MATERIALS AND METHODS}

\section{Animals}

Male C57BL/6 wild-type (WT) mice were purchased from Charles River Laboratories. The CCR6 KO mice for this study were male inbred C57BL/6 mice, purchased from the Jackson Laboratory (Bar Harbor, ME, USA). All mice were 8-9 weeks old at the start of the smoke exposure. The local Ethics
Committee for animal experimentation of the faculty of Medicine and Health Sciences (Ghent, Belgium) approved all experiments (ECD 27/07).

\section{Cigarette Smoke Exposure}

Mice were exposed to main stream cigarette smoke, as described previously. ${ }^{19}$ Briefly, groups of 10-12 mice were exposed to the tobacco smoke of five cigarettes (Reference Cigarette 3R4F without filter; University of Kentucky, Lexington, KY, USA) four times per day with a 30-min smoke-free interval, 5 days per week for 24 weeks (chronic smoke exposure). An optimal smoke:air ratio of 1:6 was obtained. The control groups were exposed to air. Carboxyhaemoglobin in serum of smoke-exposed mice reached a non-toxic level of $8.7 \pm 0.31 \%$ (compared with $0.65 \pm 0.25 \%$ in air-exposed mice), which is similar to carboxyhaemoglobin blood concentrations of human smokers. ${ }^{20}$

\section{IgA Quantification in Luminal Samples}

At $24 \mathrm{~h}$ after the last exposure, mice were weighed and killed with an overdose of pentobarbital. The abdominal cavity was opened and the small intestine was removed. Next, intestinal washes were collected by flushing the small intestine with $2 \mathrm{ml}$ of ice-cold PBS with $0.1 \%$ BSA and $1 \mathrm{mg} / \mathrm{ml}$ protease inhibitors (Protease Inhibitor Cocktail, Complete Mini, Roche, Basel, Switzerland). The collected samples were centrifuged for $10 \mathrm{~min}$ at $4^{\circ} \mathrm{C}$ and the supernatant was stored at $-80^{\circ} \mathrm{C}$ for IgA analysis. A commercially available ELISA kit (Alpha Diagnostic International, San Antonio, TX, USA) was used to determine IgA titre in intestinal washes.

\section{Single-Cell Suspensions of PP and Mesenteric Lymph Nodes}

After flushing, PP and mesenteric lymph nodes were removed from the small intestine. Samples were minced by scissors and put in digestion medium in a humidified incubator at $37^{\circ} \mathrm{C}$ and $5 \% \mathrm{CO}_{2}$ for $30 \mathrm{~min}$. Fragments were resuspended, fresh digestion medium was added and incubation at $37^{\circ} \mathrm{C}$ was continued for $15 \mathrm{~min}$. Then, samples were centrifuged and resuspended in PBS containing $10 \mathrm{mM}$ EDTA for $5 \mathrm{~min}$ at room temperature on a shaker. After red blood cell lysis, the cells were washed, passed through a $50-\mu \mathrm{m}$ strainer and kept on ice until labelling. Cell counting was performed with a Z2 Beckman Coulter particle counter (Beckman Coulter, Ghent, Belgium).

\section{Flow Cytometry of PP and Mesenteric Lymph Nodes}

Single-cell populations of mouse PP and mesenteric lymph nodes were stained with MHCII-PE-Cy5 (eBioscience Inc., San Diego, CA, USA), CD11c-PE-TexRed (Invitrogen Corp., Carlsbad, CA, USA), CD11b-Horizon V450 (BD Pharmingen, San Diego, CA, USA), CD19-AlexaFluor 700 (eBioscience), CD3-AlexaFluor 700 (eBioscience), CD4-APCCy7 (BD Pharmingen) and CD8-PE-Cy7 (eBioscience). An Aqua Live/Death marker (Invitrogen) was used to identify 
live cells. For analysis of regulatory $\mathrm{T}$ cells (Treg), use was made of anti-CD4-PE-Cy5, anti-CD25-PE-Cy7 and antiFoxP3-APC (all purchased from eBioscience). Acquisition was performed on an LSR cytometer equipped with FACSDiva software (both BD Pharmingen). The FlowJo software was used for data analysis (TreeStar Inc., Ashland, OR, USA).

\section{Quantification of Apoptosis}

Samples of PP and ileum were fixed in $4 \%$ buffered formalin, processed for paraffin embedding and eventually cut into $2-\mu \mathrm{m}$-thick sections. After antigen retrieval and blocking, primary antibody (polyclonal rabbit anti-active capsase-3; R\&D systems, Minneapolis, MN, USA) or rabbit IgG isotype control (Abcam, Cambridge, UK) was applied for $1 \mathrm{~h}$, followed by biotin-labelled goat anti-rabbit secondary antibody (DAKO, Carpinteria, CA, USA) for $30 \mathrm{~min}$ and HRP-conjugated streptavidin (DAKO) for $30 \mathrm{~min}$. AEC (3-amino-9ethyl-carbazole) was used as enzyme substrate before counterstaining with haematoxylin.

For TUNEL (terminal deoxynucleotidyl transferase dUTP nick-end labelling), the in situ cell death detection kit alkaline phosphatase (AP) of Roche was used. Staining was performed as recommended by Roche. Briefly, after pre-treatment using citrate, sections were incubated with TUNEL reaction mixture for $60 \mathrm{~min}$ at $37^{\circ} \mathrm{C}$ in a humidified dark chamber. After rinsing, Converter-AP was applied to the sections for $30 \mathrm{~min}$ in the dark. Finally, sections were incubated with substrate solution for $15 \mathrm{~min}$ in the dark at room temperature and counterstained with haematoxylin.

The apoptotic index in the FAE was determined as the percentage of apoptotic cells per 100 epithelial cells enumerated, as described by Heczko et al. ${ }^{21}$ Quantification was performed at six different levels of the PP. Quantification of apoptosis in the subepithelial dome was accomplished by means of a Zeiss KS400 image analyser platform. Quantification was performed at five different levels of the PP; the number of apoptotic cells per area of the subepithelial dome was calculated.

\section{Immunofluorescent Double Staining for UEA-1 and Caspase-3}

Fluorescent double-labelling started with antigen retrieval, followed by blocking with $1 \%$ BSA and $0.1 \%$ FSG in PBS and incubation with the rabbit anti-caspase- 3 antibody (R\&D systems) and the secondary antibody (goat anti-rabbit Alexa 596, Invitrogen). Subsequently, FITC-conjugated UEA-1 (Sigma Aldrich, St Louis, MO, USA) was applied, followed by DAPI nuclear staining. For immunofluorescent staining for UEA-1 alone, antigen retrieval and blocking was followed by incubation with FITC-conjugated UEA-1 and DAPI staining.

\section{Immunohistochemistry for CCL9 and CCL20}

Cryosections of PP were air-dried and fixed with ice-cold acetone. Endogenous peroxidase activity was quenched with $1 \% \mathrm{H}_{2} \mathrm{O}_{2}$, followed by blocking of nonspecific binding sites with $2 \%$ rabbit serum and $1 \%$ BSA in PBS. Subsequently, slides were incubated with the primary antibody (polyclonal goat anti-CCL9 and polyclonal goat anti-CCL20, both R\&D Systems) or goat IgG isotype control (Santa Cruz Biotechnology, Santa Cruz, CA, USA) for $90 \mathrm{~min}$ at $37^{\circ} \mathrm{C}$ and with the biotinylated rabbit anti-goat secondary antibody (DAKO) for $30 \mathrm{~min}$ at room temperature. Then, HRP-conjugated streptavidin (DAKO) was applied for $30 \mathrm{~min}$. AEC was used as enzyme substrate before counterstaining with haematoxylin.

\section{RNA Preparation and RT-PCR}

RNA from PP was extracted using the Qiagen RNeasy Mini Kit (Qiagen, Hilden, Germany). Subsequently, cDNA was obtained by reverse transcription of RNA with the Transcriptor First Strand cDNA synthesis kit (Roche) following manufacturer's instructions and using a 2:1 ratio of hexa:oligodT primers. Expression of target genes CCL2, CCL9, CCL19, CCL20, CCR1, CCR6, tumour necrosis factor- $\alpha$ (TNF$\alpha), I L-1 \beta, I F N-\gamma, I L-6, I L-10$ and TGF- $\beta$ and reference genes Gapdh (glyceraldehyde-3-phosphate dehydrogenase), Hprt1 (hypoxanthine phosphoribosyltransferase 1) and Tfrc (transferrin receptor) mRNA was analysed with the TaqMan Gene Expression Assays (Applied Biosystems). Real-time PCR reactions were performed in duplicate using diluted cDNA template and the LightCycler480 Probes Master (Roche). Amplifications were performed on a LightCycler480 detection system (Roche) with the following cycling conditions: $10 \mathrm{~min}$ incubation at $95^{\circ} \mathrm{C}$ and 50 cycles of $95^{\circ} \mathrm{C}$ for $10 \mathrm{~s}$ and $60^{\circ} \mathrm{C}$ for $15 \mathrm{~s}$. Expression of target genes was corrected by a normalization factor that was calculated based on the expression of three reference genes (Gapdh, Hprt1, Tfrc), using the geNorm applet according to the guidelines and theoretical framework described previously. ${ }^{22}$

\section{Statistical analysis}

Reported values are expressed as mean \pm s.e.m. (standard error of the mean) and error bars were marked as the s.e.m. Statistical analysis was performed by SPSS 16 Software (SPSS 16 Inc., Chicago, IL, USA) using Student's $t$-test for normally distributed populations and Mann-Whitney $U$-test for populations where normal distribution was not accomplished. For comparison of more than two groups, use was made of two-way analysis of variance (ANOVA), followed by post hoc least significant difference test, or non-parametric KruskalWallis test if conditions for ANOVA were not met. A $P$-value of less than 0.05 was considered significant.

\section{RESULTS \\ Cigarette Smoke Exposure Induces Apoptosis in Follicle-Associated Epithelium of PP}

To investigate whether cigarette smoke affects the FAE of PP, the apoptotic index was determined by means of immunohistochemistry for active caspase-3, a marker for apoptosis. Following smoke exposure, significantly more FAE 
cells stained positive for caspase-3 compared to air-exposed animals (Figure 1a and $\mathrm{b}$ ). This difference in apoptotic index was confirmed using TUNEL, an alternative detection method for apoptosis (data not shown). To establish whether cigarette smoke-induced apoptosis was limited to the FAE, apoptosis in the subepithelial dome was quantified as well. The number of apoptotic cells in the subepithelial dome was similar in both groups (Figure 1c and d). In contrast to FAE, the villous epithelium of the ileum did not show increased apoptosis after smoke exposure (Supplementary Figure 1A-G).

An important feature of FAE is the presence of $M$ cells, epithelial cells whose main function is transcytosis of antigens and macromolecules through the epithelial barrier into the PP, allowing generation of mucosal immune responses. First, we quantified M-cell numbers in the FAE by means of staining for UEA-1, a murine M-cell marker. Interestingly, smoke-exposed mice had a significantly lower proportion of $\mathrm{M}$ cells than air-exposed animals (Figure 2a and b). Secondly, to investigate whether smokeinduced apoptosis also affects $\mathrm{M}$ cells, we performed a fluorescent double staining for active-caspase-3 and UEA-1. In both groups, very few $\mathrm{M}$ cells showed signs of apoptosis (Figure 2c). Moreover, M-cell apoptosis did not differ between air- and smoke-exposed mice.
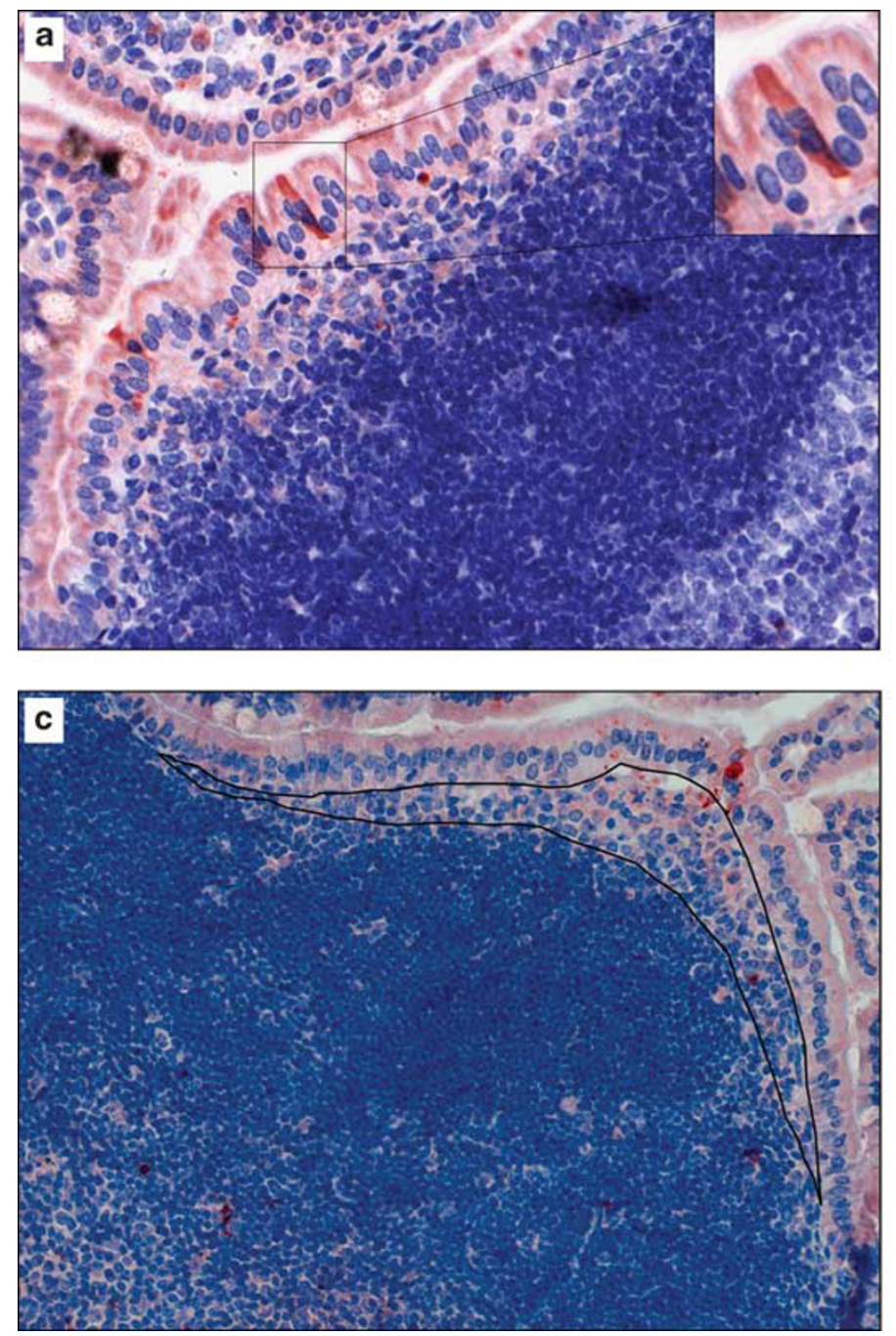

d
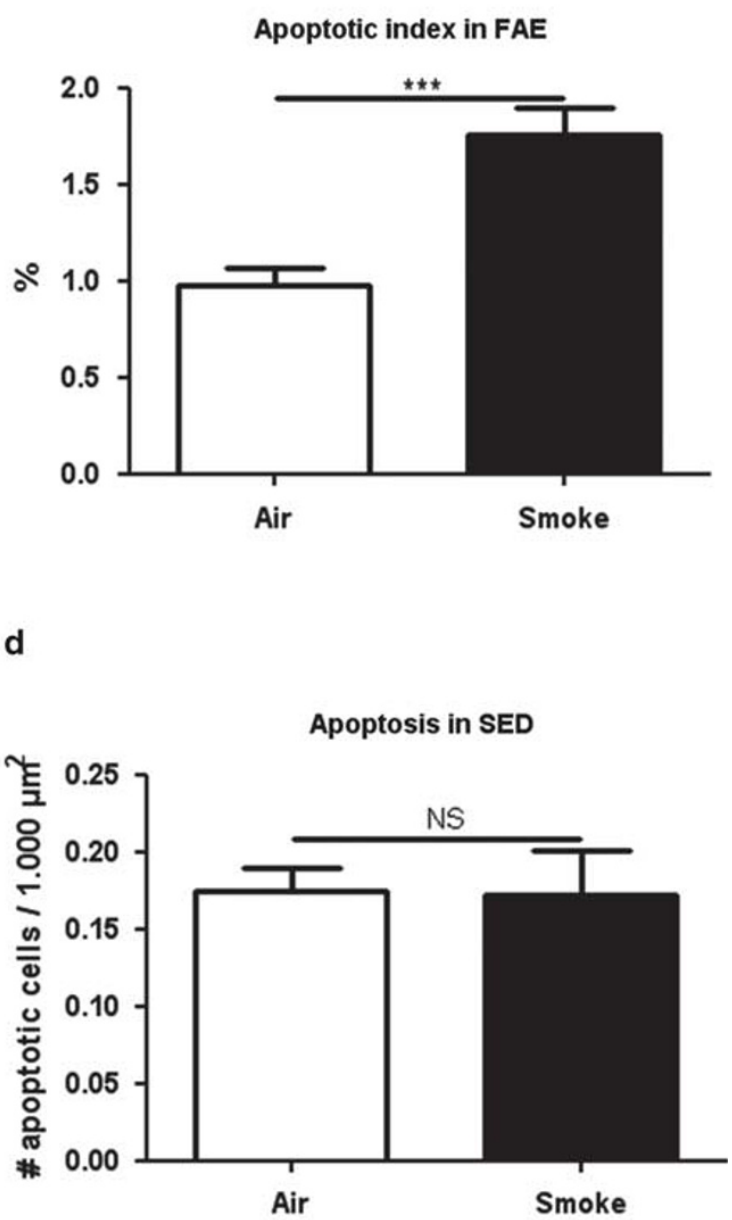

Figure 1 Smoke exposure induces apoptosis in follicle-associated epithelium (FAE) of Peyer's patches (PP). (a) Immunohistochemistry for active caspase-3 in a PP of a smoke-exposed mouse, with apoptotic cells in the FAE. Inset: details of an apoptotic cell. (b) Apoptotic index, defined as the percentage of apoptotic cells in FAE (determined by active caspase-3 immunohistochemistry), after smoke exposure (1.76 $\pm 0.14 \%$ ) compared with air exposure $(0.98 \pm 0.09 \%)$. Each group consists of 20 PP from four different mice. (c) The subepithelial dome was defined as the region of the PP between the FAE and the germinal follicles. Image analysis software calculated the number of apoptotic cells (determined by active caspase-3 immunohistochemistry) in this area. (d) Apoptotic cell number per area in the subepithelial dome. Data are from one representative of two independent experiments. Data are represented as mean \pm s.e.m. NS: nonsignificant; ${ }^{* * P} P<0.001$. 

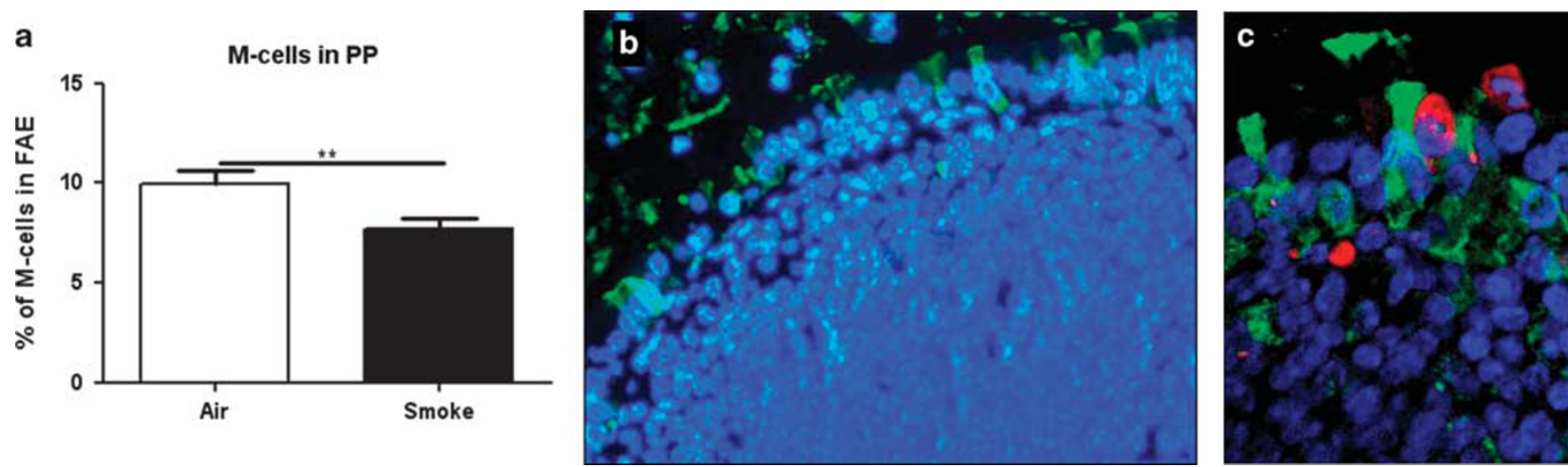

Figure 2 M-cell percentage decreases after exposure to cigarette smoke. (a) M-cell percentage, defined as the percentage of Ulex europaeus agglutinin-1 (UEA-1)-positive cells in the follicle-associated epithelium (FAE), in smoke- (7.67 $\pm 0.59 \%)$ and air-exposed animals (9.99 $\pm 0.62 \%)$. Each group consists of 20 PP from four different mice. (b) Immunofluorescent staining with UEA-1 (green) for quantification of M cells in the FAE. (c) Confocal microscopy shows absence of colocalization of active caspase-3 (red) and the M-cell marker UEA-1 (green). Data are from one representative of two independent experiments. Data are represented as mean \pm s.e.m. ${ }^{*} p<0.01$.

\section{Immune Cell Composition of PP is Influenced by Smoke Exposure}

To further investigate the influence of cigarette smoke on the GALT in the small intestine, PPs were quantified. Similar numbers were found in air- and smoke-exposed animals (5-9PP per mouse). Furthermore, size of ileal PP did not differ between both groups (Supplementary Figure 2A).

Next, the different cell populations in PP were examined by means of flow cytometry. We observed an increase in dendritic cells (DCs) in smoke-exposed animals (Figure 3a and $\mathrm{b}$ ). The rise was even more obvious in the CD11b + DC subset, which practically doubled (Figure $3 \mathrm{c}$ ).

Although the total lymphocyte number, defined as the sum of $\mathrm{CD} 3+$ and $\mathrm{CD} 19+$ cells, did not change after cigarette smoke exposure (data not shown), shifts were seen in total B-cell percentage (Supplementary Figure 2B). The T-cell percentage increased significantly after smoke exposure (Supplementary Figure 2C).

T-cell subsets were further analysed. The CD8 + T-cell subset increased significantly after smoke exposure (Supplementary Figure 2D), as well as the CD4 + T-cell subset and Treg (Figure $4 \mathrm{a}-\mathrm{c}$ ).

To examine the influence of smoke exposure on other lymphoid structures related to the small intestine, mesenteric lymph nodes were analysed. Size, determined as total cell count of three lymph nodes, did not differ between both groups. Furthermore, no difference in immune cell composition of mesenteric lymph nodes was observed between airand smoke-exposed mice (data not shown).

\section{Cigarette Smoke Exposure Suppresses IL-10 Expression in PP}

To examine whether the observed changes in immune cell composition in PP were associated with inflammation, mRNA of pro-inflammatory and anti-inflammatory cytokines were studied. No changes in expression of TNF- $\alpha$, IL- $1 \beta$, IFN- $\gamma$ or IL- 6 were detected, indicating that smoke exposure did not induce alterations in pro-inflammatory cytokine levels. Also TGF- $\beta 1$, an anti-inflammatory cytokine, did not undergo any changes in its mRNA expression. However, IL-10 expression showed a significant decrease after smoke exposure (Supplementary Figure 3A-F).

Furthermore, the lower B-cell percentage in PP prompted us to investigate the influence of cigarette smoke inhalation on mucosal IgA antibody production in the small intestine. PP are important inductive sites for IgA responses in the gut and IgA + B cells, which are precursors for IgA-producing plasma cells, are mainly generated in the $\mathrm{PP}^{23}$ Total luminal IgA immunoglobulin concentration was measured by ELISA. However, no significant differences were seen between air- and smoke-exposed mice after 24 weeks of exposure (data not shown).

\section{Expression of DC-Attracting Chemokines Increases on Cigarette Smoke Exposure}

To unravel the mechanisms leading to an increase in DC and the CD11b + DC subset in PP, mRNA expression of chemokines involved in recruitment of DC was studied. Expression of CCL9 and CCL20 increased after smoke exposure (Figure 5a and $\mathrm{b}$ ). These chemokines are known to be expressed by the FAE and attract CD11b + DC to the subepithelial dome through interaction with their respective receptors CCR1 and CCR6, both present on DC. ${ }^{24}$ In contrast, expression of CCL19, a chemokine involved in attracting DC to the interfollicular region of PP, was not altered after cigarette smoke exposure (Figure 5c). Also CCL2, a chemokine that has a role in migration and maturation of monocyte-derived DC, was not expressed differentially in airand smoke-exposed mice (Figure 5d). Furthermore, mRNA expression of the chemokine receptors CCR1 and CCR6 was determined. CCR1 expression was not influenced by cigarette smoke exposure (Figure 5e). Expression of CCR6 decreased in the smoke-exposed group compared with air-exposed mice (Figure 5f). 
a

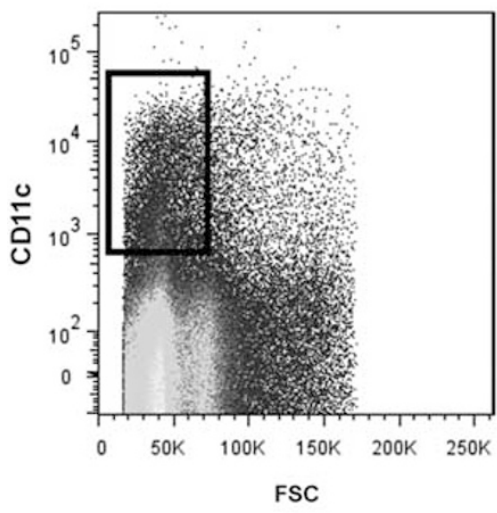

DC

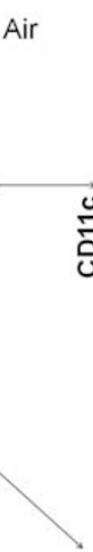

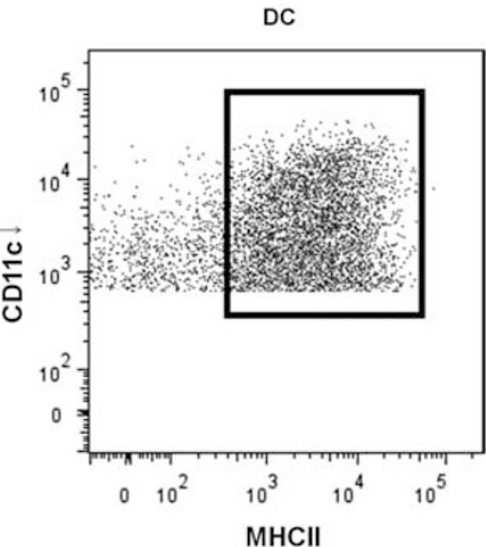
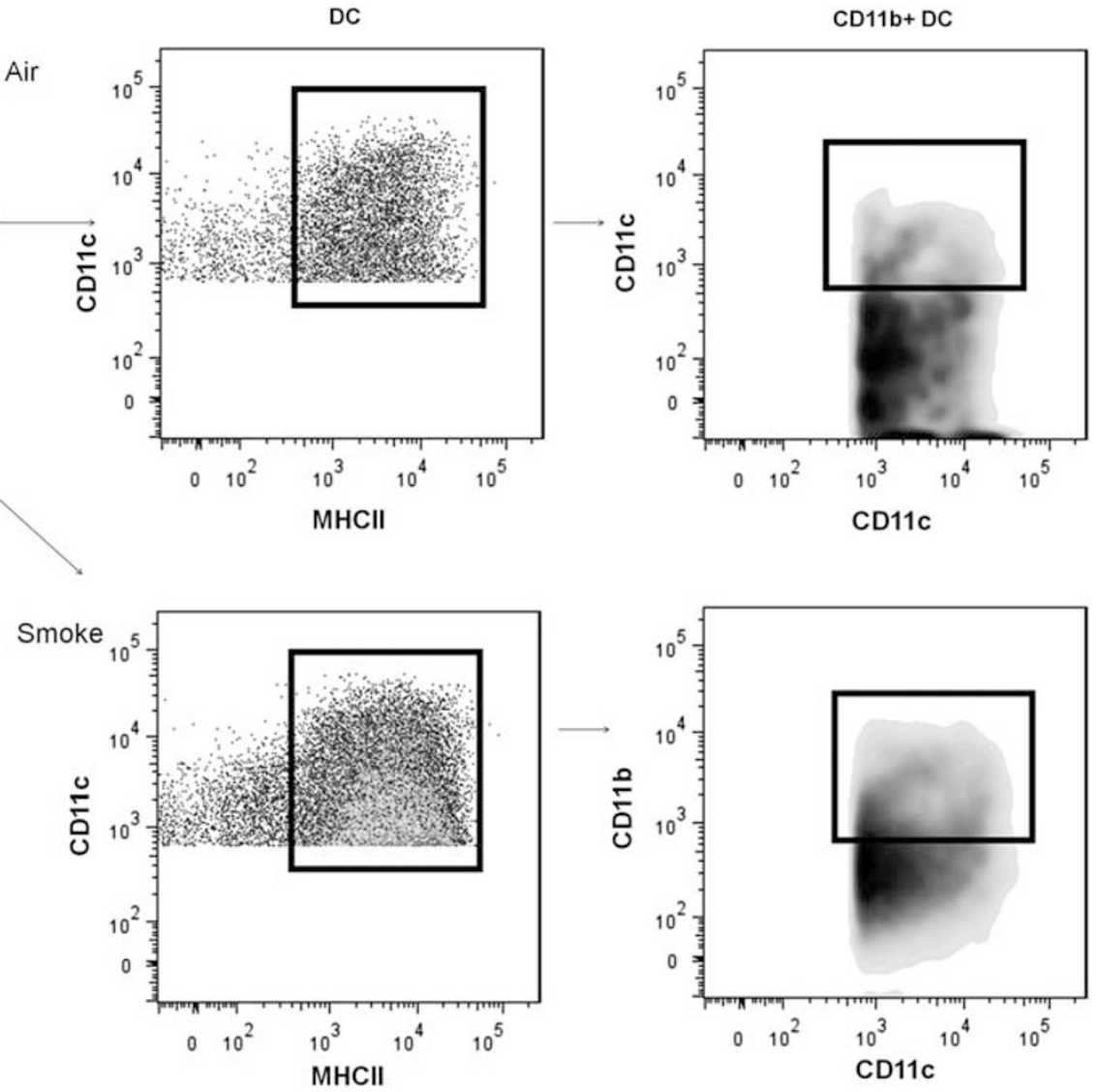

b

DC

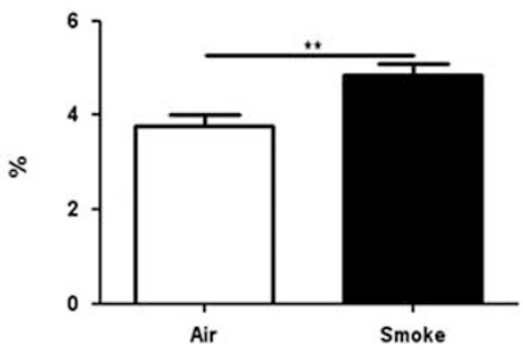

c

$C D 11 b+D C$

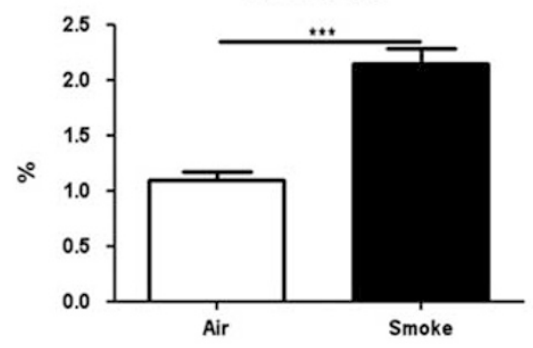

Figure 3 Cigarette smoke is associated with dendritic cell (DC) accumulation in Peyer's patches (PP). Analysis was performed on three most distal ileal PP. Results are expressed as percentage of live cells, of air- and smoke-exposed mice, respectively. (a) Gating strategy for DC in PP. DCs were defined as a CD11C highly positive and major histocompatibility class II (MHCII)-positive cell population. Within this population, CD11b + cells were identified as the CD11b + DC subset. (b) DC: $3.8 \pm 0.3$ versus $4.8 \pm 0.3 \%$. (c) CD11b + DC subset: $1.1 \pm 0.1$ versus $2.1 \pm 0.1 \%$. Data are from one representative of two independent experiments. $N=9-11$ mice per group. Data are represented as mean \pm s.e.m. ${ }^{*} P<0.01 ;{ }^{* *} P<0.001$.

Immunohistochemical staining on cryosections of PP confirmed that the FAE is the principal localization of CCL9 and CCL20 protein in PP. The staining in the FAE appeared stronger after smoke exposure compared to air exposure (Figure 6a and d).

\section{CCR6 Signalling is Not Required for Smoke-Induced Increase of DCs in PP}

Given the previously shown role of the CCL20-CCR6 axis in DC recruitment and our own observations on smoke-in- duced expression of CCL20 in PP, we repeated the chronic smoke experiment in WT versus CCR6 KO mice.

First, size of PP was compared between WT and CCR6 KO mice. PPs of CCR6 KO mice were smaller than those of WT mice, in both air- and smoke-exposed animals. Similar to PP in WT mice, smoke exposure did not affect size of PP in CCR6 KO mice (Figure 7a). Secondly, flow cytometric analysis confirmed the smoke-induced increase in $\mathrm{DC}$ and CD11b + DC percentage. The increases in CCR6 $\mathrm{KO}$ mice were comparable to those in WT mice and 
a
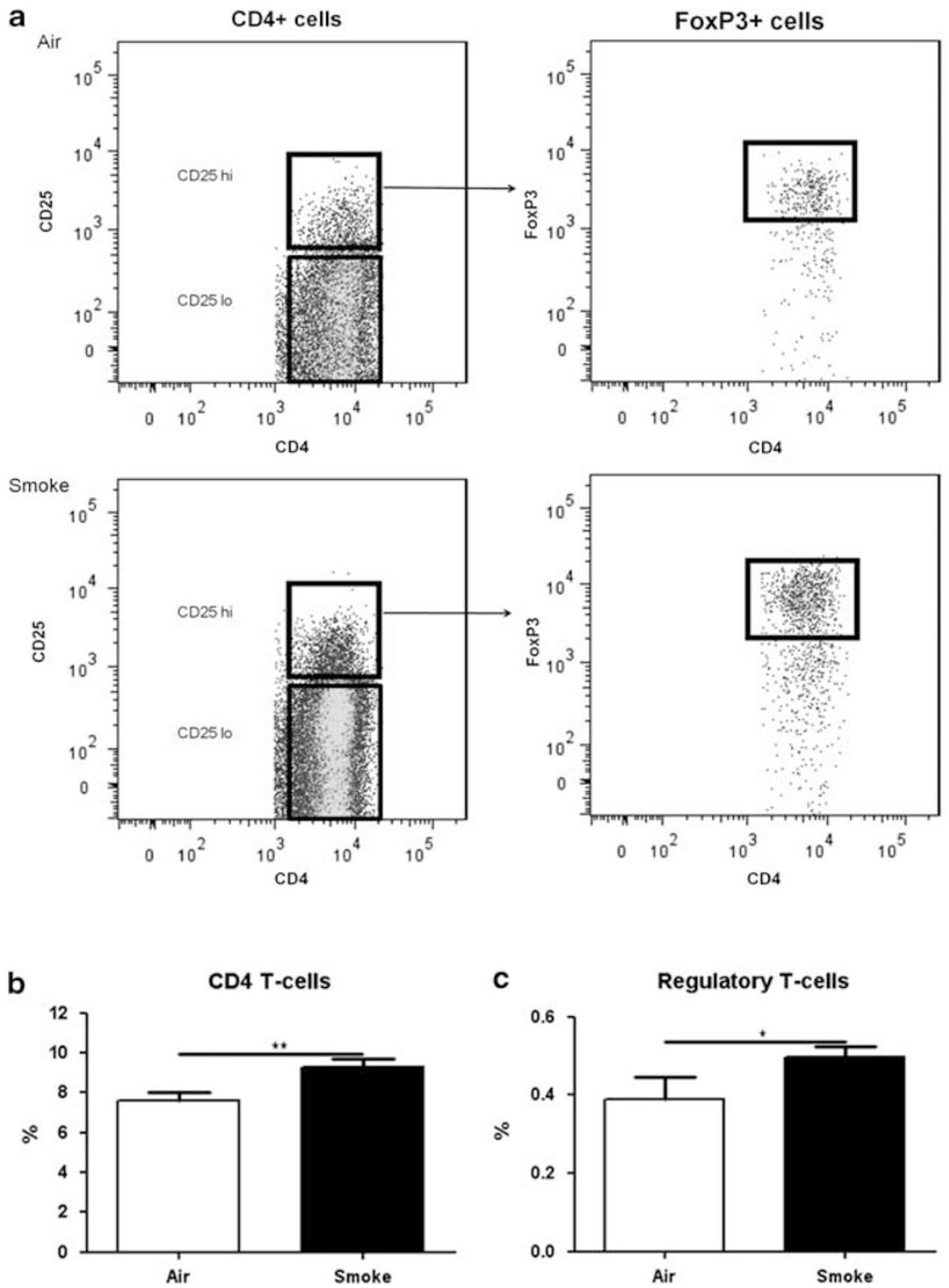

Figure 4 Cigarette smoke exposure leads to an increase in regulatory T cells (Treg) in Peyer's patches (PP). Analysis was performed on the three most distal ileal PP. Results are expressed as percentage of live cells, of air- and smoke-exposed mice, respectively. (a) Gating strategy for Treg in PP. In the population with double positivity for CD3 and CD4, Tregs were defined as FoxP3 +, CD25hi cells. (b) CD4 + T cells: $7.6 \pm 1.3$ versus $9.3 \pm 1.4 \%$. (c) Treg: $0.40 \pm 0.16$ versus $0.50 \pm 0.08 \%$. Data are from one representative of two independent experiments. $N=9-11$ mice per group. Data are represented as mean \pm s.e.m. ${ }^{\star} P<0.05 ;{ }^{* * P}<0.01$

DC percentages did not differ between WT and KO mice (Figure $7 \mathrm{~b}$ and $\mathrm{c}$ ).

\section{DISCUSSION}

In this study, we investigated the influence of chronic cigarette smoke exposure on murine gut-associated lymphoid tissue in the ileum. Our hypothesis was that smoke exposure causes changes to the epithelium and the immune cell composition of the ileal immune system, which may predispose to development of intestinal inflammation. The most striking findings were a cigarette smoke-induced increase of apoptosis in the FAE of PP, accompanied by increased recruitment of $\mathrm{DC}$, in particular the $\mathrm{CD} 11 \mathrm{~b}+\mathrm{DC}$ subset, to PP. Furthermore, the DC recruitment was accompanied by increased CD4 + (including Treg) and CD8 $+\mathrm{T}$ cells. The smoke-induced changes were present in WT mice as well as in CCR6 KO mice, indicating that the mechanism of recruitment of immune cells is not (solely) dependent on CCL20CCR6 signalling.

Smoke-induced apoptosis of epithelial cells is described in several organs and is involved in the pathogenesis of diseases like chronic obstructive pulmonary disease, peptic ulcers and age-related macular degeneration. The most plausible explanation for these findings is that smoking causes cellular injury due to oxidative stress. ${ }^{25-27}$ This is the first report that describes smoke-induced apoptosis in the intestine. It is 
a

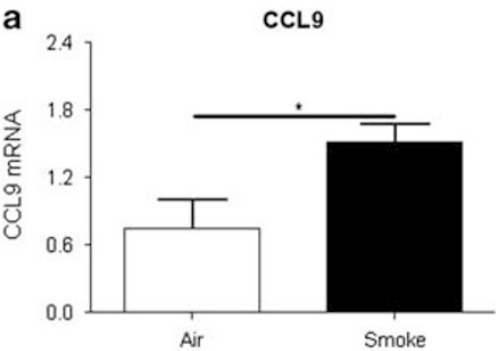

C

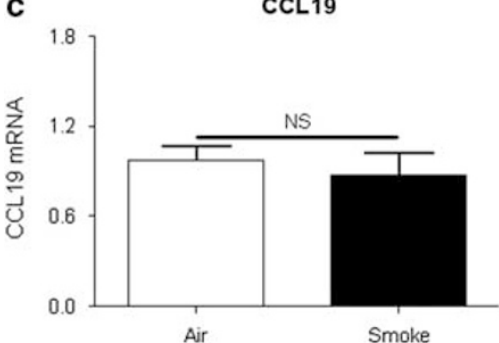

e

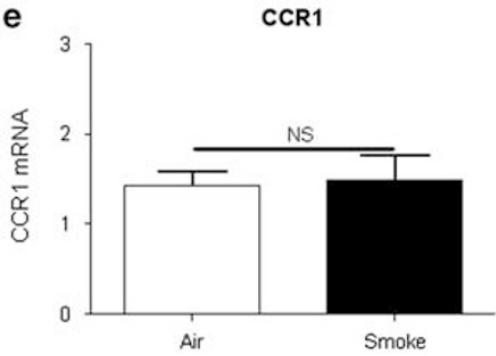

b

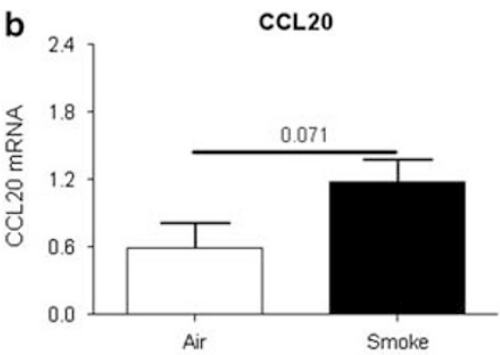

d

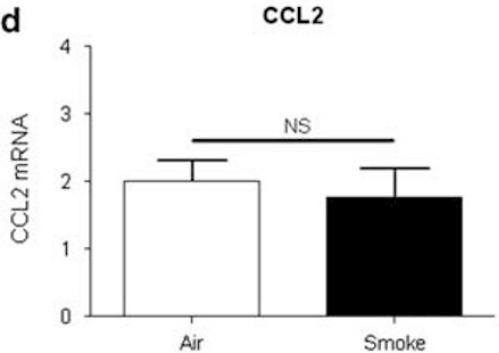

f

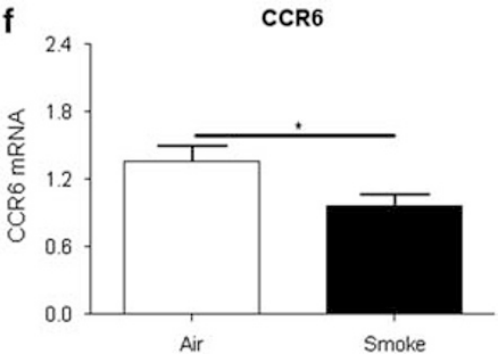

Figure 5 mRNA expression of chemokines in Peyer's patches (PP), relative to the expression of three reference genes (Hprt1 (hypoxanthine phosphoribosyltransferase 1), Gapdh (glyceraldehyde-3-phosphate dehydrogenase) and Tfrc (transferrin receptor)). $n=6$ mice per group. (a) Expression of CCL9 doubles after smoke exposure ( $0.74 \pm 0.25$ versus $1.51 \pm 0.17)$. (b) Expression of CCL20 increases from $0.59 \pm 0.21$ to $1.18 \pm 0.19$ in smoke-exposed mice. (c and d) Expression of CCL19 and CCL2 is not altered by smoke exposure. (e) Expression of CCR1 is not influenced by smoke exposure. (f) Expression of CCR6 decreases from $1.36 \pm 0.31$ to $0.96 \pm 0.25$ after smoke exposure. Data are represented as mean \pm s.e.m. $N=6$ mice per group. NS: nonsignificant; ${ }^{\star} P<0.05$.

known that small erosions are found in the FAE of patients with CD. These epithelial defects may enhance contact between luminal antigens and GALT, triggering the development of intestinal inflammation. ${ }^{28}$ We could not show erosions in the FAE of smoke-exposed mice, but it is possible that an apoptotic index almost twice as high as in air-exposed mice has implications for the epithelial barrier function. $\mathrm{M}$ cells, responsible for the transepithelial transport in FAE, did not show increased apoptosis after smoke exposure.

The second important finding in this study was the accumulation of DC to the PP, especially the CD11b + DC subset showing a marked increase. The function of this subset is uptake of antigens transported through the FAE and presentation of those antigens to CD4 + cells, which were also increased. CD11b + DC express CCR6 and are known to migrate towards CCL20, the only chemokine ligand for CCR6, which in PP is expressed predominantly by the FAE. As a result, these cells are localized in the subepithelial dome, in close vicinity to the FAE. ${ }^{29}$ Surprisingly, the increase in DC and CD11b + DC was also present in CCR6-deficient mice, indicating that CCL20-CCR6 signalling is not a prerequisite for smoke-induced recruitment of DC and that other factors must be involved. These findings are in contrast with observations in the lung, where we showed that the smokeinduced DC increase in WT mice was attenuated in CCR6 $\mathrm{KO}$ mice. $^{30}$

A higher CCL20 expression was observed in PP after smoke exposure. It is known that stressed intestinal epithelium has an increased expression of CCL20, and increased apoptosis in the FAE may contribute to a stressful environment. ${ }^{31}$ Smoke exposure also increased CCL9 expression in $\mathrm{PP}$ of smoke-exposed mice, explaining why CD11b + DC are still increased in smoke-exposed CCR6 KO mice. Similar to CCL20, CCL9 is expressed exclusively in the FAE and attracts $\mathrm{CD} 11 \mathrm{~b}+\mathrm{DC}$ to the subepithelial dome through interaction with its receptor CCR1. Indeed, earlier studies in CCR6-deficient mice showed that the CD11b + DC subset was still present in the subepithelial dome, in spite of the disruption of the CCL20-CCR6 axis. ${ }^{24}$ As both CCL9 and CCL20 are upregulated, it can be assumed that there is a functional redundancy and that both the CCL9-CCR1 and the CCL20-CCR6 pathways have a role in the increase of DC in 

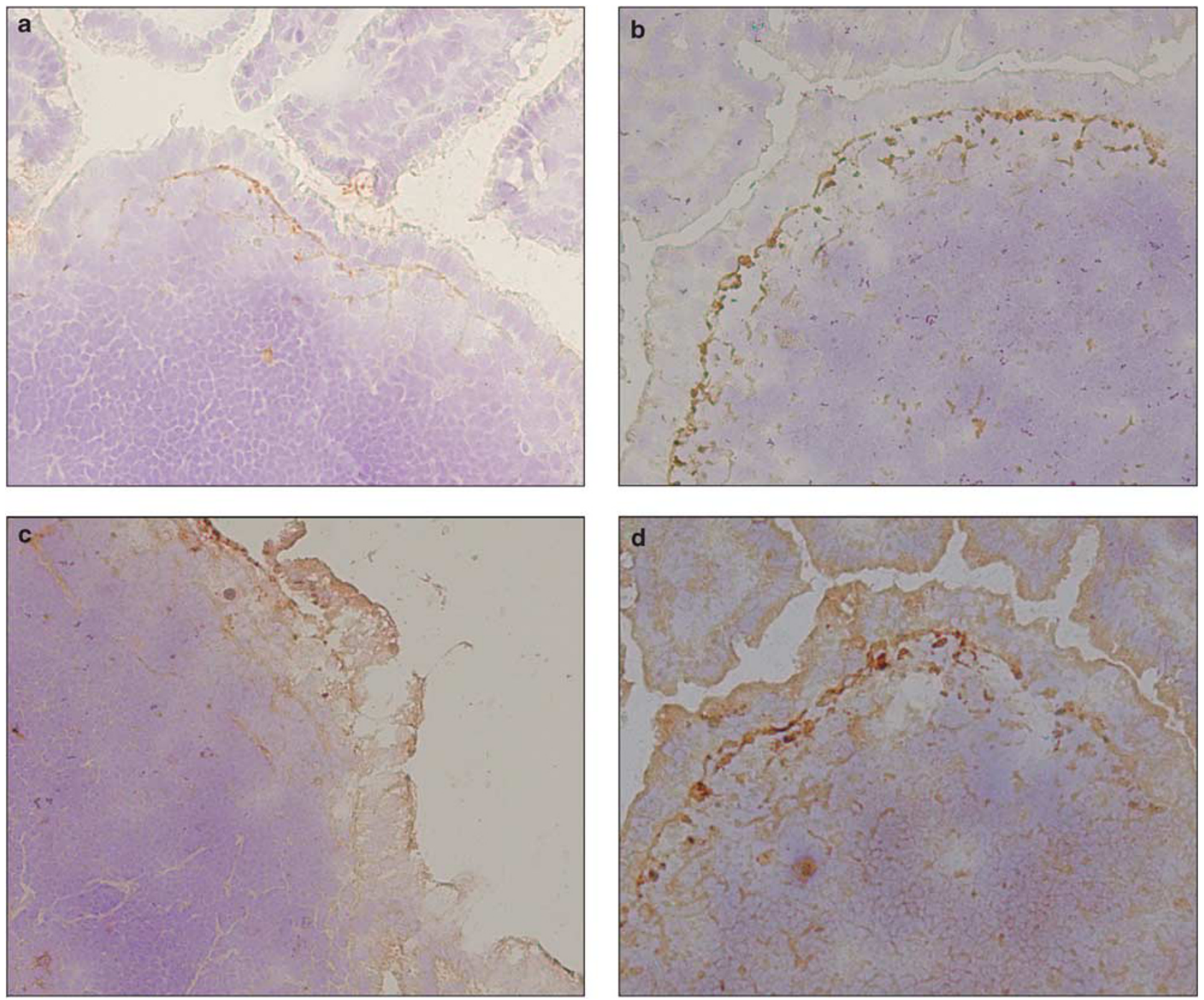

Figure 6 Protein expression of CCL9 and CCL20 in murine Peyer's patches (PP) after air and smoke exposure. (a) CCL9 expression in a PP of an air-exposed mouse. (b) CCL9 expression in a PP of a smoke-exposed mice. (c) CCL20 expression in an air-exposed mouse. (d) CCL20 expression in smoke-exposed mouse. Immunohistochemical staining for CCL9 and CCL20 was performed on cryosections of PP. All images were taken with the same magnification.
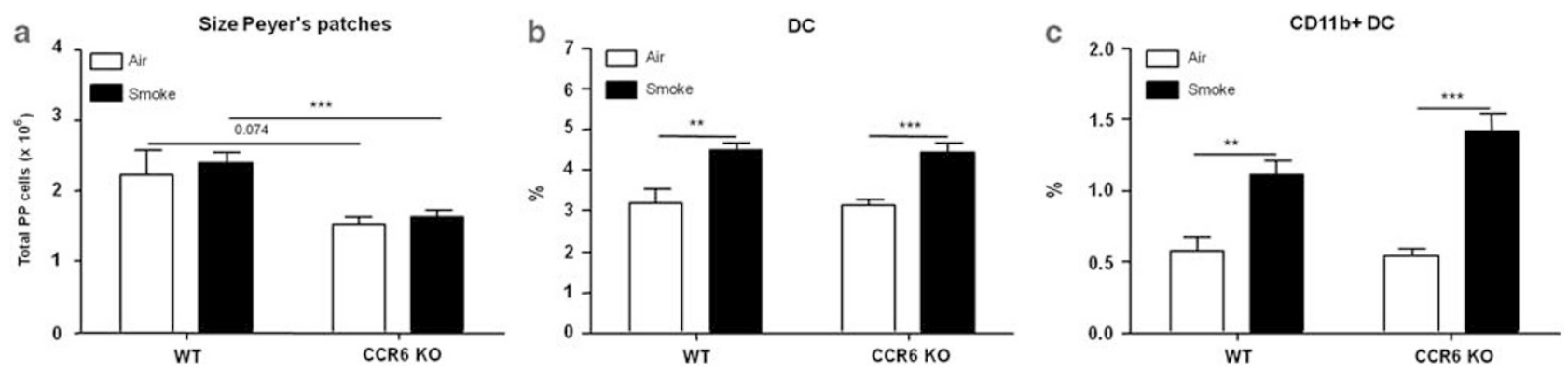

Figure 7 Impact of CCR6 on immune cell composition of Peyer's patches (PP). Analysis was performed on the three most distal ileal PP. (a) Size of PP after smoke exposure compared with air exposure, as determined by measuring the total amount of cells with a Coulter particle counter.

(b) Dendritic cell (DC) percentage in wild-type (WT) and knockout (KO) mice. (c) CD11b + DC subset in WT and KO mice. Data are represented as mean \pm s.e.m. $N=9-12$ mice per group. ${ }^{* * P}<0.01 ;{ }^{* *} P<0.001$.

PP after smoke exposure. Immunohistochemistry confirmed that the FAE is the main location for CCL9 and CCL20 proteins in PP.
In contrast to these chemokines, their respective receptors are not upregulated. CCR6 even shows a significant decrease. This can be explained by the fact that not only DC, but also 
the more abundant B-cell population expresses CCR6. ${ }^{32}$ As B cells tend to decrease after smoke exposure, it can be expected that the associated decrease in CCR6 overrules the significant but modest increase in DC.

In mesenteric lymph nodes, which-unlike PP-are not covered by FAE, these changes in DC or other immune cells were not observed, suggesting that alterations of the epithelial barrier and direct contact with luminal antigens are essential to trigger the smoke-induced immune cell accumulation.

Thirdly, we showed an increase of T cells after smoke exposure. This is not unexpected, as antigen-loaded CD11b + DC are able to induce T-cell differentiation, and differentiation of CD4 $+\mathrm{T}$ cells into Treg in particular. ${ }^{33-35}$ This process results in oral tolerance to bacterial and other antigens. Furthermore, a decrease in B cells after smoke exposure was observed, but without an accompanying change in luminal IgA production. As the majority of gut IgA + plasma cells are generated in the germinal centres of PP, it could be expected that a decrease in PP B cells is followed by a reduction in secreted IgA. A possible explanation for the maintenance of luminal IgA levels is that the function of PP $\mathrm{B}$ cells in IgA + plasma cell generation is compensated by B cells in isolated lymphoid follicles or that IgA + plasma cells are directly generated in the lamina propria of the gut. ${ }^{36,37}$

The murine model in this study has several advantages. It offers a physiological way of cigarette smoke exposure, which approximates long-term smoking in human beings. It is validated as a model to examine smoke-induced respiratory diseases. ${ }^{38}$ Although no animal model can perfectly mimic human disease, this model offers important advantages over established models for assessing smoke-induced changes in the gut. Furthermore, the use of a murine model enabled us to investigate the ileum, a region that is less evident than the colon to study in human beings, as it is less accessible and PP are often difficult to identify endoscopically. As smoking principally affects Crohn's ileitis, exploring the effect of smoking on the ileum is indispensable for understanding the role of smoking in the pathogenesis of CD.

Our aim was to investigate the effect of cigarette smoke on normal gut immunity; no inflammation was induced. The actual changes in DC and lymphocyte populations we observed were relatively small; this could be due to the fact that mice were evaluated in the absence of intestinal inflammation. In this respect, our study substantially differs from most published reports on smoking and the gut, which use models of intestinal inflammation such as IL-10 KO mice and trinitrobenzene sulphonic acid-induced colitis. ${ }^{13-16}$ It is possible that addition of inflammatory stress will amplify the observed changes in PP immune cell composition. No smoke-induced increase in inflammatory cytokines was observed in our experiment. However, expression of IL-10, an anti-inflammatory cytokine, decreased significantly after smoke exposure, possibly rendering PP more vulnerable to inflammatory stimuli.
Smoke-induced apoptosis in the epithelium covering PP may compromise the barrier function. Several studies suggest that epithelial apoptosis is an important factor in the barrier disruption associated with intestinal inflammation. ${ }^{39-41}$ However, the functional role of epithelial apoptosis in barrier dysfunction is still controversial. Bullen et $a l^{42}$ showed that epithelial cell shedding in the small intestine is associated with apoptosis, but does not cause barrier defects. Furthermore, in vitro experiments with epithelial cell lines showed that blocking apoptosis could not prevent the increase in intestinal permeability associated with exposure to inflammatory cytokines. ${ }^{43}$ Future research needs to elucidate the exact role of apoptosis in epithelial barrier dysfunction. Interestingly, we could not show increased apoptosis in the villous epithelium of the ileum after smoke exposure; hence, smoke-induced apoptosis in the ileum appears to be limited to the epithelium covering the PP. This might be because the FAE, due to its close contact with antigenic stimuli and intestinal flora, is exposed to a higher basal stress than the villous epithelium and therefore is more vulnerable to additional oxidative stress caused by smoke exposure.

We hypothesize that smoke-induced apoptosis leads to a diminished barrier function of the FAE covering PP, triggering a subsequent infiltration of antigens. This can result in immune cell attraction to the damaged region. In our model, it appears that indeed DCs accumulate to the site of increased apoptosis, possibly due to the observed upregulation of the chemokines CCL9 and CCL20. The function of this CD11b + DC subset is uptake of antigens transported by the FAE. On oral antigen presentation, CD11b + DC produce IL-10 and TGF- $\beta$, stimulating oral tolerance by induction of Treg. Therefore, this DC subset is considered as a tolerogenic cell population. However, it is suggested that upon bacterial stimulation, CD11b + DC migrate towards the interfollicular region for antigen presentation to $\mathrm{T}$ cells, inducing a Th1 response. So the function of CD11b + DC in PP may depend on the nature of the antigens they are confronted with. ${ }^{29,44}$ Furthermore, both effector T cells and Treg are increased in PP of the smoke-exposed group. We assume that these increased T-cell populations reach a new equilibrium without inducing inflammation in normal PP.

However, in predisposed individuals, such as patients at risk for developing $\mathrm{CD}$, the balance between antigen exposure and tolerance to these antigens is disrupted, for example, by an excess of T-cell effector cells or by a deficiency in regulatory mechanisms. Indeed, CD4 + and CD8 + T-cell increase in PP was observed in a mouse model for CD and an increase in both CD4 + and CD8 $+\mathrm{T}$ cells was reported in patients with active $\mathrm{CD}$, whereas the Treg population was lowered. ${ }^{45-47}$ Also, the combination of smoke-induced apoptosis with other proapoptotic factors, such as the proinflammatory cytokine TNF- $\alpha$, can account for a misbalance between immune defence and tolerance. ${ }^{48}$ Once this imbalance reaches a threshold, tolerance to oral antigens is lost, 
resulting in an uncontrolled immune response and intestinal inflammation.

In summary, this study is the first to investigate the effect of prolonged smoke exposure on the ileal GALT in a murine model. We show that smoke exposure leads to increased epithelial apoptosis in the FAE and to accumulation of $\mathrm{CD} 11 \mathrm{~b}+\mathrm{DC}$ in PP, probably by the upregulation of CCL9 and CCL20, and to an increase in T cells. Taken together, these results suggest that smoking causes alterations in the epithelial barrier of PPs, leading to changes in the composition of the underlying immune cell population. In normal individuals, a new balance between immune activation and tolerance is established. However, in combination with other predisposing factors, these alterations may lead to the development of the intestinal inflammation distinctive for $\mathrm{CD}$. Our findings can help to understand the pathogenesis of $\mathrm{CD}$ in smokers.

Supplementary Information accompanies the paper on the Laboratory Investigation website (http://www.laboratoryinvestigation.org)

\section{DISCLOSURE/CONFLICT OF INTEREST}

The authors declare no conflict of interest.

\section{ACKNOWLEDGEMENTS}

We thank Philip Vlummens, Harald Peeters, Debby Laukens, Nico Boon and Tom van de Wiele for the helpful discussions. We are grateful to Hamida Hammad for providing the confocal images. We thank Isabelle Rottiers and Katrien De Visschere for the technical help with the ELISA and immunohistochemistry, Dorothea van Limbergen and Ran Rumes for the support with the animal experiments and the processing of the samples, and Eliane Castrique, Christelle Snauwaert, Marie-Rose Mouton, Katleen De Saedeleer, Anouk Goethals, Ann Neesen, Indra De Borle and Greet Barbier en Evelien Spruyt for the excellent technical support. This work was supported by Special Research Fund of Ghent University (01J17507) and Concerted Research Action of Ghent University (BOF 10/GOA/021). Stephanie Verschuere is supported by a doctoral grant from the Special Research Fund of Ghent University (01D21009). Ken Bracke is a postdoctoral researcher of the Fund for Scientific Research (FWO) in Flanders.

1. Loftus Jr EV, Sandborn WJ. Epidemiology of inflammatory bowel disease. Gastroenterol Clin N Am 2002;31:1-20.

2. Loftus CG, Loftus Jr EV, Harmsen WS, et al. Update on the incidence and prevalence of Crohn's disease and ulcerative colitis in Olmsted County, Minnesota, 1940-2000. Inflamm Bowel Dis 2007;13:254-261.

3. Lapidus A. Crohn's disease in Stockholm County during 1990-2001: an epidemiological update. World J Gastroenterol 2006;12:75-81.

4. Xavier RJ, Podolsky DK. Unravelling the pathogenesis of inflammatory bowel disease. Nature 2007;448:427-434.

5. Birrenbach T, Bocker U. Inflammatory bowel disease and smoking: a review of epidemiology, pathophysiology, and therapeutic implications. Inflamm Bowel Dis 2004;10:848-859.

6. Cottone M, Rosselli M, Orlando A, et al. Smoking habits and recurrence in Crohn's disease. Gastroenterology 1994;106:643-648.

7. Lakatos PL, Szamosi T, Lakatos L. Smoking in inflammatory bowel diseases: good, bad or ugly? World J Gastroenterol 2007;13:6134-6139.

8. Karban A, Eliakim R. Effect of smoking on inflammatory bowel disease: is it disease or organ specific? World J Gastroenterol 2007;13: 2150-2152.

9. Arnott ID, Williams N, Drummond $\mathrm{HE}$, et al. Whole gut lavage fluid interleukin-1 beta and interleukin- 8 in smokers and non-smokers with Crohn's disease in clinical remission. Dig Liver Dis 2002;34:424-429.
10. Sher ME, Bank S, Greenberg R, et al. The influence of cigarette smoking on cytokine levels in patients with inflammatory bowel disease. Inflamm Bowel Dis 1999;5:73-78.

11. Nielsen $\mathrm{OH}$, Bjerrum JT, Csillag $\mathrm{C}$, et al. Influence of smoking on colonic gene expression profile in Crohn's disease. PLoS One 2009;4:e6210.

12. Aldhous $M C$, Prescott RJ, Roberts $S$, et al. Does nicotine influence cytokine profile and subsequent cell cycling/apoptotic responses in inflammatory bowel disease? Inflamm Bowel Dis 2008;14:1469-1482.

13. Sun YP, Wang HH, He Q, et al. Effect of passive cigarette smoking on colonic alpha7-nicotinic acetylcholine receptors in TNBS-induced colitis in rats. Digestion 2007;76:181-187.

14. Galeazzi F, Blennerhassett PA, Qiu B, et al. Cigarette smoke aggravates experimental colitis in rats. Gastroenterology 1999;117:877-883.

15. Guo X, Ko JK, Mei QB, et al. Aggravating effect of cigarette smoke exposure on experimental colitis is associated with leukotriene $B(4)$ and reactive oxygen metabolites. Digestion 2001;63:180-187.

16. Eliakim R, Fan QX, Babyatsky MW. Chronic nicotine administration differentially alters jejunal and colonic inflammation in interleukin-10 deficient mice. Eur J Gastroenterol Hepatol 2002;14:607-614.

17. Zijlstra FJ, Srivastava ED, Rhodes $M$, et al. Effect of nicotine on rectal mucus and mucosal eicosanoids. Gut 1994;35:247-251.

18. van der Strate BW, Postma DS, Brandsma CA, et al. Cigarette smokeinduced emphysema: a role for the B cell? Am J Respir Crit Care Med 2006;173:751-758.

19. D'hulst Al, Vermaelen KY, Brusselle GG, et al. Time course of cigarette smoke-induced pulmonary inflammation in mice. Eur Respir J 2005;26:204-213.

20. Macdonald G, Kondor N, Yousefi V, et al. Reduction of carboxyhaemoglobin levels in the venous blood of cigarette smokers following the administration of carbogen. Radiother Oncol 2004;73:367-371.

21. Heczko U, Carthy CM, O'Brien BA, et al. Decreased apoptosis in the ileum and ileal Peyer's patches: a feature after infection with rabbit enteropathogenic Escherichia coli 0103. Infect Immun 2001;69: 4580-4589.

22. Vandesompele J, De Preter K, Pattyn F, et al. Accurate normalization of real-time quantitative RT-PCR data by geometric averaging of multiple internal control genes. Genome Biol 2002;3:RESEARCH0034.

23. Fagarasan S, Honjo T. Regulation of IgA synthesis at mucosal surfaces. Curr Opin Immunol 2004;16:277-283.

24. Zhao X, Sato A, Dela Cruz CS, et al. CCL9 is secreted by the follicleassociated epithelium and recruits dome region Peyer's patch CD11b+ dendritic cells. J Immunol 2003;171:2797-2803.

25. Ma L, Wang HY, Chow JY, et al. Cigarette smoke increases apoptosis in the gastric mucosa: role of epidermal growth factor. Digestion 1999;60:461-468.

26. Kim HP, Wang $\mathrm{X}, \mathrm{Chen} \mathrm{ZH}$, et al. Autophagic proteins regulate cigarette smoke-induced apoptosis: protective role of heme oxygenase-1. Autophagy 2008;4:887-895.

27. Fujihara M, Nagai N, Sussan TE, et al. Chronic cigarette smoke causes oxidative damage and apoptosis to retinal pigmented epithelial cells in mice. PLoS One 2008;3:e3119.

28. Fujimura $Y$, Kamoi R, lida M. Pathogenesis of aphthoid ulcers in Crohn's disease: correlative findings by magnifying colonoscopy, electron microscopy, and immunohistochemistry. Gut 1996;38:724-732.

29. Iwasaki A, Kelsall BL. Localization of distinct Peyer's patch dendritic cell subsets and their recruitment by chemokines macrophage inflammatory protein (MIP)-3alpha, MIP-3beta, and secondary lymphoid organ chemokine. J Exp Med 2000;191:1381-1394.

30. Bracke KR, $\mathrm{D}^{\prime}$ Hulst $\mathrm{Al}$, Maes $\mathrm{T}$, et al. Cigarette smoke-induced pulmonary inflammation and emphysema are attenuated in CCR6deficient mice. J Immunol 2006;177:4350-4359.

31. Tanaka $Y$, Imai $T$, Baba $M$, et al. Selective expression of liver and activation-regulated chemokine (LARC) in intestinal epithelium in mice and humans. Eur J Immunol 1999;29:633-642.

32. McDonald KG, MCDonough JS, Wang C, et al. CC chemokine receptor 6 expression by $\mathrm{B}$ lymphocytes is essential for the development of isolated lymphoid follicles. Am J Pathol 2007;170:1229-1240.

33. Min SY, Park KS, Cho ML, et al. Antigen-induced, tolerogenic CD11c+, $\mathrm{CD} 11 \mathrm{~b}+$ dendritic cells are abundant in Peyer's patches during the induction of oral tolerance to type II collagen and suppress experimental collagen-induced arthritis. Arthritis Rheum 2006;54:887-898. 
34. Nagatani $\mathrm{K}$, Komagata $\mathrm{Y}$, Asako $\mathrm{K}$, et al. Antigen-specific regulatory $T$ cells are detected in Peyer's patches after the interaction between $T$ cells and dendritic cells which is loaded with orally administered antigen. Immunobiology 2010.

35. Iwasaki A, Kelsall BL. Unique functions of CD11b+, CD8 alphat, and double-negative Peyer's patch dendritic cells. J Immunol 2001;166: 4884-4890.

36. Yamamoto $M$, Rennert $P, M c G h e e ~ J R$, et al. Alternate mucosal immune system: organized Peyer's patches are not required for $\lg A$ responses in the gastrointestinal tract. J Immunol 2000;164:5184-5191.

37. Uematsu S, Fujimoto $\mathrm{K}$, Jang $\mathrm{MH}$, et al. Regulation of humoral and cellular gut immunity by lamina propria dendritic cells expressing Tolllike receptor 5. Nat Immunol 2008;9:769-776.

38. Brusselle GG, Bracke KR, Maes T, et al. Murine models of COPD. Pulm Pharmacol Ther 2006;19:155-165.

39. Vereecke L, Sze M, Guire CM, et al. Enterocyte-specific A20 deficiency sensitizes to tumor necrosis factor-induced toxicity and experimental colitis. J Exp Med 2010;207:1513-1523.

40. Zeissig S, Bojarski C, Buergel N, et al. Downregulation of epithelial apoptosis and barrier repair in active Crohn's disease by tumou necrosis factor alpha antibody treatment. Gut 2004;53:1295-1302.
41. Schulzke JD, Ploeger $S$, Amasheh $M$, et al. Epithelial tight junctions in intestinal inflammation. Ann N Y Acad Sci 2009;1165:294-300.

42. Bullen TF, Forrest $\mathrm{S}, \mathrm{Campbell} F$, et al. Characterization of epithelial cell shedding from human small intestine. Lab Invest 2006;86:1052-1063.

43. Bruewer $M$, Luegering $A$, Kucharzik $T$, et al. Proinflammatory cytokines disrupt epithelial barrier function by apoptosis-independent mechanisms. J Immunol 2003;171:6164-6172.

44. Sato A, Iwasaki A. Peyer's patch dendritic cells as regulators of mucosal adaptive immunity. Cell Mol Life Sci 2005;62:1333-1338.

45. Biswas A, Liu YJ, Hao L, et al. Induction and rescue of Nod2-dependent Th1-driven granulomatous inflammation of the ileum. Proc Natl Acad Sci USA 2010;107:14739-14744.

46. Muller S, Lory J, Corazza N, et al. Activated CD4+ and CD8+ cytotoxic cells are present in increased numbers in the intestinal mucosa from patients with active inflammatory bowel disease. Am J Pathol 1998;152:261-268.

47. Chamouard P, Monneaux F, Richert Z, et al. Diminution of Circulating CD4+CD25 high T cells in naive Crohn's disease. Dig Dis Sci 2009;54 2084-2093.

48. Izcue A, Coombes $J$, Powrie F. Regulatory lymphocytes and intestinal inflammation. Annu Rev Immunol 2009;27:313-338. 